\title{
A MODEL OF BRILL-NOETHER THEORY FOR RANK TWO VECTOR BUNDLES AND ITS PETRI MAP *
}

\author{
TAN XIAO-JIANG ${ }^{\dagger}$
}

\begin{abstract}
We study here the Brill-Noether theory for rank two vector bundles. First we construct a parameter space $H_{d}$ for all base point free rank two vector bundles of degree $d$ which generated by its sections. Then for each $E \in H_{d}$, we define a $2 g \times d$ matrix $W_{E}$ for which we call it the Brill-Noether matrix of $E$, it shares the same properties as the Brill-Noether matrix $W_{D}$ for effective divisor $D$. By using $W_{E}$, the Brill-Noether variety $C_{2, d}^{r}=\left\{E \in H_{d} \mid \operatorname{dim} H^{0}(C, E) \geq r+1\right\}$ could be given by $C_{2, d}^{r}=\left\{E \in H_{d} \mid \operatorname{rank}\left(W_{E}\right) \leq d-r+1\right\}$, so $C_{2, d}^{r}$ is a determinant variety, we get its expected dimension is $4(g-1)+1-(r+1)(2(g-1)-d+r+1)+2 r+1$. On the other hand, by using $W_{E}$, we define the Petri map to be $P: H^{0}(C, K(-E)) \otimes \operatorname{Im}\left\{H^{0}(C, E) \mapsto H^{0}(C,[D])\right\} \mapsto$ $H^{0}(C, K[D](-E))$, we show that $C_{2, d}^{r}$ has the expected dimension if and only if the Petri map is injective.
\end{abstract}

1. Introduction . Let $\mathrm{C}$ be a smooth irreducible complex projective curve of genus $g(\mathrm{C}$ a Compact Riemann surface $), L$ a line bundle on $\mathrm{C}$. We also use $L$ to denote the sheaf of holomorphic sections of $L$. The Brill-Noether theory for line bundles is to study those bundles $L$ for which both $H^{0}(C, L)$ and $H^{1}(C, L)$ are non-zero $(L$ is then called special line bundle).

Let $C_{d}$ be the $d$-fold symmetric product of $\mathrm{C}, C_{d}$ is a d-dimensional complex manifold. It is the space of all effective divisors of degree d. Since each line bundle $L$ with $H^{0}(C, L) \neq 0$ is defined by an effective divisor, so $C_{d}$ could be considered as a parameter space for all line bundles $L$ with $\operatorname{deg}(L)=d$ and $H^{0}(C, L) \neq 0$.

Define on $C_{d}$ the Brill-Noether variety $C_{d}^{r}$ to be

$$
C_{d}^{r}=\left\{D \in C_{d} \mid \operatorname{dim} H^{0}(C,[D]) \geq r+1\right\} .
$$

Where $[D]$ is the line bundle defined by divisor $\mathrm{D}$.

$C_{d}^{r}$ could be considered as a parameter space for line bundles $L$ with $\operatorname{deg}(L)=d$ and $\operatorname{dim} H^{0}(C, L) \geq r+1$. The key tool to study $C_{d}^{r}$ is the Brill-Noether matrix.

Let $D=n_{1} p_{1}+\cdots+n_{k} p_{k}$ be a given effective divisor with $d=\operatorname{deg}(D)=$ $n_{1}+\cdots+n_{k}$. For $i=1, \cdots, k$, let $z_{i}$ be a local coordinate at $p_{i}$ with $z_{i}\left(p_{i}\right)=0$. Let $\left\{w_{1}, \cdots, w_{g}\right\}$ be a linear basis of the space of all holomorphic forms on $\mathrm{C}$, for each i assume at $p_{i}, w_{t}\left(z_{i}\right)=f_{t i}\left(z_{i}\right) d z_{i}$ for $t=1, \cdots, g$, let $W_{D}$ be the matrix of the restrictions of $\left\{w_{1}, \cdots, w_{g}\right\}$ on $\mathrm{D}$, that is

$$
\begin{aligned}
& W_{D}=\left[\begin{array}{cc}
w_{1} & \mid D \\
w_{2} & \mid D \\
\vdots \\
w_{g} & \left.\right|_{D}
\end{array}\right] \\
& =\left[\begin{array}{ccccccc}
f_{11}\left(p_{1}\right) & \cdots & \frac{1}{\left(n_{1}-1\right) !} f_{11}^{\left(n_{1}-1\right)}\left(p_{1}\right) & f_{12}\left(p_{2}\right) & \cdots & \frac{1}{\left(n_{2}-1\right) !} f_{12}^{\left(n_{2}-1\right)}\left(p_{2}\right) & \cdots \\
\vdots & \ddots & \vdots & \vdots & \ddots & \vdots & \vdots \\
f_{g 1}\left(p_{1}\right) & \cdots & \frac{1}{\left(n_{k}-1\right) !} f_{g 1}^{\left(n_{1}-1\right)}\left(p_{1}\right) & f_{g 2}\left(p_{2}\right) & \cdots & \frac{1}{\left(n_{2}-1\right) !} f_{g 2}^{\left(n_{2}-1\right)}\left(p_{2}\right) & \cdots
\end{array}\right] .
\end{aligned}
$$

*Received February 9, 2003; accepted for publication July 5, 2003. Project supported by National Natural Science Foundation of China.

${ }^{\dagger}$ School of Mathematical Sciences, Peking University, Beijing 100871, P.R.China (tan@math.pku.edu.cn). 
For a collection of Laurent tails $\mu=\left\{\mu_{i}=\sum_{k=-n_{i}}^{-1} b_{i k} z_{i}^{k}\right\}$, we denote it as a d-dimensional vector

$$
\mu=\left(b_{1-1}, b_{1-2}, \cdots, b_{1-n_{1}}, b_{2-1}, \cdots, b_{2-n_{2}}, \cdots\right) \in C^{d} .
$$

Then $\mu$ is the Laurent of a global meromorphic function if and only if $W_{D} \cdot \mu^{t}=0$. From this one can get Riemann-Roch theorem easily.

The matrix $W_{D}$ is called the Brill-Noether matrix of $\mathrm{D}$.

Now let $\left.[D]\right|_{D}$ be the skyscraper sheaf of the restriction of $[D]$ on $\mathrm{D}$, then what we have above could be represented as

$$
\operatorname{Ker}\left(W_{D}\right)=\left\{\mu \mid \mu \in C^{d}, W_{D} \cdot \mu^{t}=0\right\} \cong \operatorname{Im}\left\{H^{0}(X,[D]) \mapsto H^{0}\left(X,\left.[D]\right|_{D}\right)\right\}
$$

and in particular, we get

$$
\operatorname{dim} H^{0}(X,[D])=\operatorname{deg}(D)-\operatorname{rank}\left(W_{D}\right)+1 .
$$

so $C_{d}^{r}$ could be defined by

$$
C_{d}^{r}=\left\{D \in C^{d} \mid \operatorname{Rank}\left(W_{D}\right) \leq d-r\right\} .
$$

It is a subvariety of $C_{d}$ which locally is defined by the simultaneously vanishing of all ( $d-$ $r+1) \times(d-r+1)$ minors of $W_{D}(\operatorname{Ref}[\mathrm{ACGH}] \mathrm{p} 159)$.

Now let $M(m, n)=M$ be the variety of all $m \times n$ complex matrices, and for $0 \leq k \leq$ $\min \{m, n\}$, denote by $M_{k}(m, n)=M_{k}$ the locus of matrices of rank at most $\mathrm{k}$, that is

$$
M_{k}=\{E \in M(m, n) \mid \operatorname{Rank}(E) \leq k\} .
$$

$M_{k}$ is an irreducible subvariety of $M(m, n)$, and $\operatorname{codim}\left(M_{k}\right)=(n-k)(m-k)(\operatorname{Ref}$ [ACGH] p67).

By using the Brill-Noether matrix, locally we have a holomorphic map $B N: C_{D} \rightarrow$ $M(m, n)$ with $B N(D)=W_{D}$ for each $D \in C_{d}$. $C_{d}^{r}$ is then could be given by $C_{d}^{r}=$ $B N^{-1}\left(M_{d-r}\right)$. From the Theory of determinant variety, we get that if $C_{d}^{r} \neq \emptyset$, then $\operatorname{codim}\left(C_{d}^{r}\right) \leq \operatorname{codim}\left(M_{d-r}\right)=(g-(d-r))(d-(d-r))$. So if $C_{d}^{r} \neq \emptyset$, then

$$
\operatorname{dim} C_{d}^{r} \geq d-r(g-d+r)=g-(r+1)(g-d+1)=\rho(g, d, r)+r .
$$

where $\rho(g, d, r)=g-(r+1)(g-d+r)$ is the Brill-Noether number for line bundles. (Ref [ACGH] p215).

It was conjectured by Brill-Noether and Proved by Griffiths-Harris [GH] that for generic $\mathrm{C}, C_{d}^{r}$ do have the expected dimension $\rho(g, d, r)+r$.

On the other hand, by study the tangent map of $B N: C_{D} \rightarrow M(m, n), D \mapsto W_{D}$, Petri got that the variety $C_{d}^{r}$ is smooth and has the "expected dimension" $\rho(g, d, r)+r$ at $D \in C_{d}^{r}-C_{d}^{r+1}$ if and only if the cup product homomorphism

$$
\mu: H^{0}(C,[D]) \otimes H^{0}(C, K[-D]) \mapsto H^{0}(C, K)
$$

is injective, where, $K$ is the canonical line bundle of $\mathrm{C}(\operatorname{Ref}[\mathrm{ACGH}] \mathrm{p} 163)$.

The map $\mu$ is called the Petri map. Again, it was proved by Gieseker[G] that for generic $\mathrm{C}$, the cup product homomorphism $\mu$ is indeed injective. This gives another prove of the result of Griffiths-Harris.

In this paper, we are trying to generalize those ideals to the study of rank two vector bundles.

First we will define a parameter space $H_{d}$ for all base point free rank two vector bundles of degree $d$ which generated by its sections( we called such vector bundles the effective vector bundles). $H_{d}$ is a d-dimensional holomorphic vector bundle on $C_{d}$, so it is a 2 d-dimensional complex manifold. 
For each $E \in H_{d}$, we construct a $2 g \times d$ matrix $W_{E}$ for $E$ which we call it the BrillNoether matrix of $E$, it shares the same properties for $E$ as the Brill-Noether matrix $W_{D}$ for line bundle $[D]$. In particular, we have

$$
\operatorname{dim} H^{0}(C, E)=d-\operatorname{Rank}\left(W_{E}\right)+2 .
$$

From this, the Brill-Noether variety of rank two vector bundles

$$
C_{2, d}^{r}=\left\{E \in H_{d} \mid \operatorname{dim} H^{0}(C, E) \geq r+1\right\}
$$

could be given by

$$
C_{2, d}^{r}=\left\{E \in H_{d} \mid \operatorname{Rank}\left(W_{E}\right) \leq d-r+1\right\} .
$$

This defines $C_{2, d}^{r}$ as a subvariety of $H_{d}$.

Also by using $W_{E}$, locally we get a holomorphic map

$$
B N: H_{d} \mapsto M(2 d, g) ; B N(E)=W_{E},
$$

so $C_{2, d}^{r}=B N^{-1}\left(M_{d-r+1}\right)$, and from the theory of determinant variety, we get that if $C_{2, d}^{r} \neq \emptyset$ then

$$
\operatorname{codim}_{2, d}^{r} \leq(2 g-(d-r+1))(d-(d-r+1))
$$

so if $C_{2, d}^{r} \neq \emptyset$, then

$$
\begin{gathered}
\operatorname{dim} C_{2, d}^{r} \geq 2 d-(2 g-(d-r+1))(d-(d-r+1))=2 d-(r+1)(2(g-1)-d+r+1)= \\
2 d-(r+1)(2(g-1)-d+r+1)+2(2(g-1)-d+r+1)= \\
4(g-1)+1-(r+1)(2(g-1)-d+r+1)+2 r+1=\rho_{2}(g, d, r)+2 r+1
\end{gathered}
$$

here $\rho_{2}(g, d, r)=4(g-1)+1-(r+1)(2(g-1)-d+r+1)$ is the Brill-Noether number for rank two vector bundles.

Also, by studying the tangent map of $B N: H_{d} \mapsto M(2 g, d)$, we generalize the Petri map to rank two vector bundles. This is for each $E \in C_{2, d}^{r}$, we define a cup product homomorphism

$$
P: H^{0}(C, K(-E)) \otimes \operatorname{Im}\left\{H^{0}(C, E) \mapsto H^{0}(C,[D])\right\} \mapsto H^{0}(C, K[D](-E)) .
$$

Here $[D]=E / I$ is the quotient bundle of $\mathrm{E}$ with respect to the trivial line bundle $I$. We call $P$ the Petri map for rank two vector bundles, and we show that $C_{2, d}^{r}$ has the "expected dimension" $\rho_{2}(g, d, r)+2 r+1$ if and only if the Petri map $P$ is injective.

\section{The parameter space $H_{d}$.}

Definition 1. A point $p \in C$ is called a base point of vector bundle $E$ if $s(p)=0$ for all $s \in H^{0}(C, E) . E$ is said to be base point free if $E$ don't have base point.

Definition 2 [A]. A rank two vector bundle $E$ is said to be generated by its sections, if $E$ has a splitting

$$
0 \mapsto L_{1} \mapsto E \mapsto L_{2} \mapsto 0
$$

such that both $H^{0}\left(C, L_{1}\right)$ and $\operatorname{Im}\left\{H^{0}(C, E) \mapsto H^{0}\left(C, L_{2}\right)\right\}$ are not zero. Where $L_{1}$ is a line sub-bundle of $E$, and $L_{2}=E / L_{1}$. 
The Brill-Noether theory for rank two vector bundles is to study those bundles $E$ with both $H^{0}(C, E)$ and $H^{1}(C, E)$ are non-zero. $E$ is then called special rank two vector bundle. If $E$ has a base point $p$, then $E \otimes[-p]$ is also special and we have $\operatorname{dim} H^{0}(C, E \otimes[-p])=$ $\operatorname{dim} H^{0}(C, E), \operatorname{dim}^{1}(C, E \otimes[-p])=\operatorname{dim}^{1}(C, E)+2$ and $\operatorname{deg}(E \otimes[-p])=\operatorname{deg}(E)-2$. We can reduce the degree of $E$. If $E$ is not generated by its sections, since $H^{0}(C, E) \neq 0$, let $s \in H^{0}(C, E)$ with $s \neq 0$, let $L_{1}$ be the line sub-bundle of $E$ which generated by $s$, $L_{2}=E / L_{1}$. Since $E$ is not generated by its sections, so $H^{0}(C, E)=H^{0}\left(C, L_{1}\right)$, the study of $H^{0}(C, E)$ could be reduced to the study of $H^{0}\left(C, L_{1}\right)$, that is reduced to the study of Brill-Noether for line bundles. So to study the Brill-Noether for rank two vector bundles, we can restrict ourself to the study of base point free vector bundles which generated by its sections.

LEMMA 1. If $E$ is a base point free rank two vector bundle which generated by its sections, then the trivial line bundle $I$ is a line sub-bundle of $E$.

Proof. This is a special case of Lemma 1.1 of [TE].

Let $E$ be a base point free rank two vector bundles which generated by its sections, assume $\operatorname{deg}(E)=d$, by our Lemma, $I$ is a line sub-bundle of $E$, so $E$ has a splitting

$$
0 \mapsto I \mapsto E \mapsto L \mapsto 0
$$

where $L=E / I$. Since $E$ is generated by its sections, we have $\operatorname{Im}\left\{H^{0}(C, E) \mapsto H^{0}(C, L)\right\} \neq$ 0 . Choose $s \in \operatorname{Im}\left\{H^{0}(C, E) \mapsto H^{0}(C, L)\right\}$ with $s \neq 0$, let $D=\operatorname{div}(s)$, then $D \geq 0$, and $L=[D] . E$ is then an extension of $[D]$ by $I$, it is determined by an element $e \in H^{1}(C,[-D])$. Since $s \in H^{0}(C,[D])$ can be lift to a section of $E$, we get in particular that $s \cdot e=0$, and from sequence

$$
\left.0 \mapsto[-D] \mapsto^{\cdot s} I \mapsto I\right|_{D} \mapsto 0
$$

we get an exact sequence

$$
0 \mapsto H^{0}(C,[-D]) \mapsto H^{0}(C, I) \mapsto H^{0}\left(C,\left.I\right|_{D}\right) \mapsto H^{1}(C,[-D]) \mapsto \cdots
$$

$s \cdot e=0$ if and only if $e \in \operatorname{Im}\left\{H^{0}\left(C,\left.I\right|_{D}\right) \mapsto H^{1}(C,[-D])\right\}$. Let $e$ be the image of some $f \in H^{0}\left(C,\left.I\right|_{D}\right), f$ is then determined uniquely up to a constant. So from $E$ we get a triple $\{I, D, f\}$.

Conversely, if we have a triple $\{I, D, f\}$, where $D$ is an effective divisor of degree $d$, and $f \in H^{0}\left(C,\left.I\right|_{D}\right)$, then let $e \in H^{1}(C,[-D])$ be the image of $f$ in the map $H^{0}\left(C,\left.I\right|_{D}\right) \mapsto$ $H^{1}(C,[-D])$ which induced from sequence $(* * *)$, let $E$ be the extension of $[D]$ by $I$ which determined by $e$, then $E$ has a splitting $0 \mapsto I \mapsto E \mapsto[D] \mapsto 0$, and $s \in \operatorname{Im}\left\{H^{0}(C, E) \mapsto\right.$ $\left.H^{0}(C,[D])\right\}$, where $s$ is the canonical section of $D\left(s \in H^{0}(C,[D])\right.$, with $\left.\operatorname{div}(s)=D\right)$. We get a base point free rank two vector bundle $E$ of degree $d$ which generated by its sections.

So to give a base point free rank two vector bundle of degree $d$ which generated by its sections will be the same as to give a triple $\{I, D, f\}$, here $D \in C_{d}$ and $f \in H^{0}\left(C,\left.[D]\right|_{D}\right)$, or the same the set of all base point free rank two vector bundle of degree $d$ which generated by its sections could be represented by the set of all triples $\{I, D, f\}$. We will denote this as $E=\{I, D, f\}$.

Now let $H_{d}$ be the vector bundle on $C_{d}$ which for each $\left.D \in \hat{C_{d}, H_{d}}\right|_{D}=H^{0}\left(C,\left.I\right|_{D}\right)$, by using local coordinate, it is easy to see that $H_{d}$ is a holomorphic vector bundle of dimension $d$ on $C_{d}$.

Each point of $H_{d}$ could be represented as a triple $E=\{I, D, f\}$, and each triple $E=$ $\{I, D, f\}$ could be represented as a point in $H_{d}$, so $H_{d}$ could be considered as a parameter space for the set of all base point free rank two vector bundles of degree $d$ which generated by its sections. 
3. Brill-Neother matrix for $E=\{I, D, f\}$. Let $L$ be a line bundle, $D=$ $n_{1} p_{1}+\cdots+n_{k} p_{k} \geq 0$ be a given effective divisor of degree $d$. For $i=1, \cdots, k$, let $z_{i}$ be a local coordinate at $p_{i}$ with $z_{i}\left(p_{i}\right)=0$. Then each $f \in H^{0}\left(C,\left.L\right|_{D}\right)$ could be represented as a set of polynomials $f=\left\{f_{i}\left(z_{i}\right)\right\}_{i=1}^{k}$, where $f_{i}\left(z_{i}\right)=a_{0}^{i}+a_{1}^{i} z_{i}+\cdots+a_{n_{i}-1}^{i} z_{i}^{n_{i}-1}$ is a polynomial of $z_{i}$ of degree less than $n_{i}$. So $f$ could also be denoted as a d-dimensional vector $f=\left(a_{0}^{1}, a_{1}^{1}, \cdots, a_{n_{1}-1}^{1} ; a_{0}^{2}, a_{1}^{2}, \cdots, a_{n_{2}-1}^{2} ; \cdots \cdots\right)$. This gives $H^{0}\left(C,\left.L\right|_{D}\right) \cong C^{d}$, where $d=\operatorname{deg}(d)$.

Definition 3. Let $L_{1}, L_{2}$ be two line bundles, $D=n_{1} p_{1}+\cdots+n_{k} p_{k} \geq 0$ be a given effective divisor. For $f=\left\{f_{i}\left(z_{i}\right)\right\}_{i=1}^{k} \in H^{0}\left(C,\left.L_{1}\right|_{D}\right)$ and $g=\left\{g_{i}\left(z_{i}\right)\right\}_{i=1}^{k} \in H^{0}\left(C,\left.L_{2}\right|_{D}\right)$, we define $f * g \in H^{0}\left(C,\left.L_{1} \otimes L_{2}\right|_{D}\right)$ to be

$$
f * g=\left\{f_{i}\left(z_{i}\right) g_{i}\left(z_{i}\right)\left(\bmod \left(z_{i}^{n_{i}}\right)\right)\right\}_{i=1}^{k} .
$$

LEMma 2. $f * g=g * f$, and $(f * g) * h=f *(g * h)$.

Proof. Trivial.

Lemma 3. For $E=\{I, D, f\}$, a section $s \in H^{0}(C,[D])$ could be lift to be a section of $H^{0}(C, E)$ ( which means $s \in \operatorname{Im}\left\{H^{0}(C, E) \mapsto H^{0}(C,[D])\right\}$ ), if and only if

$$
\left.s\right|_{D} * f \in \operatorname{Im}\left\{H^{0}(C,[D]) \mapsto H^{0}\left(C,\left.[D]\right|_{D}\right)\right\}
$$

Proof. See $[\mathrm{T}]$.

Now let $\left(w_{1}, \cdots, w_{g}\right)$ be a linear basis of $H^{0}(C, K)$ of the space of all holomorphic forms on $C$. then for effective divisor $D$, the Brill-Noether matrix $W_{D}$ for $D$ could be defined by

$$
W_{D}=\left[\begin{array}{c}
w_{1} \mid D \\
w_{2} \mid D \\
\vdots \\
\left.w_{g}\right|_{D}
\end{array}\right]
$$

An element $t \in H^{0}\left(C,\left.[D]\right|_{D}\right)$ is in the image of map $H^{0}(C,[D]) \mapsto H^{0}\left(C,\left.[D]\right|_{D}\right)$, if and only if

$$
W_{D} * t=\left[\begin{array}{c|c}
w_{1} & \left.\right|_{D} * t \\
w_{2} & \left.\right|_{D} * t \\
\vdots & \\
w_{g} & \left.\right|_{D} * t
\end{array}\right]=0
$$

That is $\operatorname{Im}\left\{H^{0}(C,[D]) \mapsto H^{0}\left(C,\left.[D]\right|_{D}\right)\right\}=\operatorname{Ker}\left\{W_{D}\right\}$.

Now for $E=\{I, D, f\}$, we define its Brill-Noether matrix $W_{E}$ to be

$$
W_{E}=\left[\begin{array}{c}
\left.w_{1}\right|_{D} \\
\left.w_{2}\right|_{D} \\
\vdots \\
\left.w_{g}\right|_{D} \\
\left.w_{1}\right|_{D} * f \\
w_{2} \mid D * f \\
\vdots \\
\left.w_{g}\right|_{D} * f
\end{array}\right]=\left[\begin{array}{c}
W_{D} \\
W_{D} * f
\end{array}\right]
$$

Theorem 1. $\operatorname{Ker}\left\{W_{E}\right\}=\left\{v \in C^{d} \mid W_{E} \cdot v=0\right\} \cong \operatorname{Im}\left\{H^{0}(C, E) \mapsto H^{0}(C,[D]) \mapsto\right.$ $\left.H^{0}\left(C,\left.[D]\right|_{D}\right)\right\}$. 
Proof. By $H^{0}\left(C,\left.[D]\right|_{D}\right) \cong C^{d}$, each $v \in C^{d}$ could be identified to an element $v \in$ $H^{0}\left(C,\left.[D]\right|_{D}\right)$, let $W_{D}$ be the Brill-Noether matrix for $D$, then $W_{D} \cdot v=W_{D} * v$, and $\left(W_{D} * f\right) * v=W_{D} *(f * v)$. So $W_{E} \cdot v=0$ if and only if $W_{D} * v=0$ and $W_{D} *(f * v)=0$. From $W_{D} * v=0$, we get that $v \in \operatorname{Im}\left\{H^{0}(C,[D]) \mapsto H^{0}\left(C,\left.[D]\right|_{D}\right)\right\}$. Let it be the image of some $s \in H^{0}(C,[D])$, this is $v=\left.s\right|_{D}$. Then from $\left(W_{D} * f\right) * v=0$, we get $\left.\left(W_{D} * f\right) * s\right|_{D}=$ $W_{D} *\left(\left.f * s\right|_{D}\right)=0$. That means $\left.f * s\right|_{D} \in \operatorname{Im}\left\{H^{0}(C,[D]) \mapsto H^{0}\left(C,\left.[D]\right|_{D}\right)\right\}$. By our Lemma $3, s$ is then can be lift to a section of $E$.

Conversely, if $v \in \operatorname{Im}\left\{H^{0}(C, E) \mapsto H^{0}(C,[D]) \mapsto H^{0}\left(C,\left.[D]\right|_{D}\right)\right\}$, let it be the image of some $s \in H^{0}(C,[D])$, so $W_{D} \cdot v=0$, and since s can be lift to a section of $\mathrm{E}$, by our Lemma $3, f * v \in \operatorname{Im}\left\{H^{0}(C,[D]) \mapsto H^{0}\left(C,\left.[D]\right|_{D}\right)\right\}$, so $W_{D} * f * v=0$, we get $W_{E} \cdot v=0$. This completes the proof.

Now from the exact sequence

$$
0 \mapsto I \mapsto E \mapsto[D] \mapsto 0
$$

we get exact sequence

$$
0 \mapsto H^{0}(C, I) \mapsto H^{0}(C, E) \mapsto H^{0}(C,[D]) \mapsto H^{1}(C, I) \mapsto \cdots
$$

Since $\operatorname{dim} H^{0}(C, I)=1$, so

$$
\begin{gathered}
\operatorname{dim} H^{0}(C, E)=\operatorname{dim} \operatorname{Im}\left\{H^{0}(C, E) \mapsto H^{0}(C,[D])\right\}+1= \\
\operatorname{dim} \operatorname{Im}\left\{H^{0}(C, E) \mapsto H^{0}(C,[D]) \mapsto H^{0}\left(C,\left.[D]\right|_{D}\right)\right\}+2= \\
\operatorname{dimKer}\left(W_{E}\right)+2=d-\operatorname{rank}\left(W_{E}\right)+2 .
\end{gathered}
$$

That is

Theorem 2. Let $E=\{I, D, F\}$ and $W_{E}$ be its Brill-Noether matrix, then we have $\operatorname{Ker}\left(W_{E}\right) \cong \operatorname{Im}\left\{H^{0}(C, E) \mapsto H^{0}(C,[D]) \mapsto H^{0}\left(C,\left.[D]\right|_{D}\right)\right\}$, and in particular $\operatorname{dim} H^{0}(C, E)=d-\operatorname{rank}\left(W_{E}\right)+2$.

Now we define the Brill-Noether variety $C_{2, d}^{r}$ for rank two vector bundles to be

$$
C_{2, d}^{r}=\left\{E \in H_{d} \mid \operatorname{dim}^{0}(C, E) \geq r+1\right\} .
$$

By Theorem 2, $C_{2, d}^{r}$ could also be given by

$$
C_{2, d}^{r}=\left\{E \in H_{d} \mid \operatorname{rank}\left(W_{E}\right) \leq d-r+1\right\} .
$$

This gives $C_{2, d}^{r}$ as a subvariety of $H_{d}$ which $C_{2, d}^{r}$ is defined locally by the simultaneously vanishing of all $(d-r+2) \times(d-r+2)$ minors of $W_{E}$.

By using the Brill-Noether matrix $W_{E}$, locally, we get a holomorphic map $B N: H_{d} \mapsto$ $M(2 g, d)$ with $B N(E)=W_{E}$ for each $E \in H_{d}$, where $M(2 g, d)$ is the variety of all $2 g \times d$ complex matrices. Let

$$
M_{d-r+1}=\{E \in M(2 g, d) \mid \operatorname{rank}(E) \leq d-r+1\} .
$$

Then $M_{d-r+1}$ is a subvariety of $\mathrm{M}(2 \mathrm{~g}, \mathrm{~d})$, and $\operatorname{codim}\left(M_{d-r+1}\right)=(2 g-(d-r+1)) \times(d-(d-$ $r+1)$ ). By definition, we have $C_{2, d}^{r}=B N^{-1}\left(M_{d-r+1}\right)$. So from the Theory of determinant variety, we get that if $C_{2, d}^{r} \neq \emptyset$, then

$$
\operatorname{codim} C_{2, d}^{r} \leq(2 g-(d-r+1)) \times(d-(d-r+1)) .
$$

This is

$$
\operatorname{dim} C_{2, d}^{r} \geq 2 d-(2 g-(d-r+1)) \times(d-(d-r+1))=
$$




$$
4(g-1)+1-(r+1)(2(g-1)-d+r+1)+2 r+1=\rho_{2}(g, d, r)+2 r+1 .
$$

Here $\rho_{2}(g, d, r)=4(g-1)+1-(r+1)(2(g-1)-d+r+1)$ is the Brill-Noether number for rank two vector bundles. Same as the case of line bundles, we get that the expected dimension of $C_{2, d}^{r}$ is $\rho_{2}(g, d, r)+2 r+1$, this is

THEOREM 3. If $C_{2, d}^{r} \neq \emptyset$, then each component of $C_{2, d}^{r}$ will have dimension at least $\rho_{2}(g, d, r)+2 r+1$.

4. The Petri map. Since $C_{2, d}^{r}=B N^{-1}\left(M_{d-r+1}\right)$, to get the dimension of $C_{2, d}^{r}$, analogous to the case of line bundles, we should consider the tangent map

$$
B N^{*}: T_{E} \mapsto T_{B N(E)}
$$

for each $E=\{I, f, D\} \in H_{d}$. Here $T_{E}$ and $T_{B N(E)}$ are the tangent space of $E$ and $B N(E)$ in $H_{d}$ and $M(2 g, d)$.

Now let $E=\{I, D, f\}$, then

$$
B N(E)=W_{E}=\left[\begin{array}{c}
W_{D} \\
W_{D} * f
\end{array}\right]
$$

Since for each $D \in C_{d}$, the tangent space of $C_{d}$ at $D$ is $T_{D}=H^{0}\left(C,\left.[D]\right|_{D}\right)$ (Ref [ACGH] $\mathrm{P} 160)$, so by definition we get that the tangent space of $H_{d}$ at $E$ is $T_{E}=H^{0}(C,[D] \mid D$ )$\oplus H^{0}\left(C,\left.I\right|_{D}\right)$.

Now let $t=(-v, u) \in T_{E}=H^{0}\left(C,\left.[D]\right|_{D}\right) \oplus H^{0}\left(C,\left.I\right|_{D}\right)$, then by direct calculation, we have

$$
B N^{*}(t)=\left[\begin{array}{c}
\dot{W}_{D} *(-v) \\
\dot{W}_{D} *(-v) * f+W_{D} * u
\end{array}\right]
$$

Where $\dot{W}_{D}$ means the differential of $W_{D}$ with respect to the local coordinates, and $\dot{f}=I$.

To get the dimension of $C_{2, d}^{r}$, we need to get the dimension of the space $V=\left\{t \in T_{E} \mid\right.$ $\left.B N^{*}(t) \in T_{B N(E)}\left(M_{d-r+1}\right)\right\}$. But from the theory of determinant variety(Ref [ACGH] p69), we know that $B N^{*}(t) \in T_{B N(E)}\left(M_{d-r+1}\right)$ if and only if $\operatorname{Ker}\left(W_{E}\right) \cdot B N^{*}(t) \subset \operatorname{Im}\left(W_{E}\right)=$ $C^{2 g} \cdot W_{E}$. Here $\operatorname{Ker}\left(W_{E}\right)=\left\{(b, e)=\left(b_{1}, \cdots, b_{g} ; e_{1}, \cdots, e_{g}\right) \in C^{d} \mid(b, e) W_{E}=0\right\}$.

Now let $(b, e)=\left(b_{1}, \cdots, b_{g} ; e_{1}, \cdots, e_{g}\right) \in \operatorname{Ker}\left(W_{E}\right)$, this is $(b, e) \cdot W_{E}=b \cdot W_{D}+e$. $W_{D} * f=0$. Choose an open cover $\left\{U_{\alpha}\right\}_{\alpha=1}^{k}$ of $C$, let $s=\left\{s_{\alpha}\right\}_{\alpha=1}^{k} \in H^{0}(C,[D])$ be the canonical section of $[D]$, this is $s \in H^{0}(C,[D])$ and $\operatorname{div}(s)=D$. for the linear basis $\left\{w_{1}, \cdots, w_{g}\right\}$ of the holomorphic forms, let $w_{i}$ be given with respect to the open cover by $w_{i}=\left\{w_{\alpha i}\right\}$, let $b w=b_{1} w_{1}+\cdots+b_{g} w_{g}=\left\{b_{1} w_{\alpha 1}+\cdot+b_{g} w_{\alpha g}\right\}=\left\{b w_{\alpha}\right\} \in H^{0}(C, K)$, and $e w=e_{1} w_{1}+\cdots+e_{g} w_{g}=\left\{e_{1} w_{\alpha 1}+\cdot+e_{g} w_{\alpha g}\right\}=\left\{e w_{\alpha}\right\} \in H^{0}(C, K)$, let $f=\left\{f_{\alpha}\right\}$ be a given representation for $f \in H^{0}\left(C,\left.I\right|_{D}\right)$, where $f_{\alpha}$ is a holomorphic function on $U_{\alpha}$.

Lemma $4 .(b, e) \in \operatorname{Ker}\left(W_{E}\right)$ if and only if

$$
F=\left\{F_{\alpha}=\left[\begin{array}{c}
e \cdot w_{\alpha} \\
-\left(b \cdot w_{\alpha}+e \cdot w_{\alpha} * f_{\alpha}\right) / s_{\alpha}
\end{array}\right]\right\} \in H^{0}(C, K(-E)) .
$$

Here $(-E)$ is the dual vector bundle of $E$.

Proof. For later using and also for making our notations easy to understand, we will give a proof of this Lemma in detail, and we will also use the proof to give a proof of Riemann-Roch Theorem for rank two vector bundles.

Let $\left\{U_{\alpha}\right\}_{\alpha=1}^{k}$ be the open cover of C. Then on $U_{\alpha} \cap U_{\beta}$, the transition matrix of $E=$ $\{I, f, D\}$ can be given by

$$
E_{\alpha \beta}=\left[\begin{array}{cc}
1 & \left(f_{\alpha}-f_{\beta}\right) / s_{\beta} \\
0 & s_{\alpha} / s_{\beta}
\end{array}\right]
$$


where $e=\left\{e_{\alpha \beta}=\left(f_{\alpha}-f_{\beta}\right) / s_{\beta}\right\}$ is a representation of $e \in H^{1}(C,[-D])$.

From $E_{\alpha \beta}$, and by the definition of dual vector bundle, the transition matrix of $K(-E)$ can be given on $U_{\alpha} \cap U_{\beta}$ by

$$
(K(-E))_{\alpha \beta}=\left[\begin{array}{cc}
k_{\alpha \beta} & 0 \\
-k_{\alpha \beta}\left(f_{\alpha}-f_{\beta}\right) / s_{\beta} & k_{\alpha \beta} s_{\beta} / s_{\alpha}
\end{array}\right]
$$

where $\left\{k_{\alpha \beta}\right\}$ is the transition function of the canonical line bundle $K$.

By definition, $K(-E)$ is an extension of $K$ by K[-D], which determined also by $f \in$ $H^{0}\left(C,\left.I\right|_{D}\right)$.

Now let $(b, e) \in \operatorname{Ker}\left(W_{E}\right)$, that is $b \cdot W_{D}+e \cdot W_{D} * f=0$, let $e w=e_{1} w_{1}+\cdots+e_{g} w_{g} \in$ $H^{0}(C, K), b w=b_{1} w_{1}+\cdots+b_{g} w_{g} \in H^{0}(C, K)$, then $b \cdot W_{D}+e \cdot W_{D} * f=0$ means ew $\left.\right|_{D} * f=-\left.b w\right|_{D}$, by our Lemma 3(also Ref [T]), that means, ew can be lift to a section of $K(-E)$ and

$$
F=\left\{F_{\alpha}=\left[\begin{array}{c}
e \cdot w_{\alpha} \\
-\left(b \cdot w_{\alpha}+e \cdot w_{\alpha} * f_{\alpha}\right) / s_{\alpha}
\end{array}\right]\right\} \in H^{0}(C, K(-E)) .
$$

is one of the lift. This can also be proved by direct computation that $F_{\alpha}=K(-E)_{\alpha \beta} \cdot F_{\beta}$.

Conversely, let

$$
F=\left\{F_{\alpha}=\left[\begin{array}{c}
e \cdot w_{\alpha} \\
v_{\alpha}
\end{array}\right]\right\} \in H^{0}(C, K(-E)) .
$$

then $e w=e_{1} w_{1}+\cdots+e_{g} w_{g}=\left\{e w_{\alpha}=\left.e_{1} w_{1}\right|_{U_{\alpha}}+\cdots+\left.e_{g} w_{g}\right|_{U_{\alpha}}\right\}$, is a section of $K$, here $e=\left(e_{1}, \cdots, e_{g}\right)$, and $F$ is a lift of ew. ew $\in H^{0}(C, K)$ can be lift to a section of $H^{0}(C, K(-E))$, by our Lemma 3 , there exists an $b w=b_{1} w_{1}+\cdots+b_{g} w_{g} \in H^{0}(C, K)$, such that $\left.e w\right|_{D} * f=-\left.b w\right|_{D}$, or the same, ew $\left.\right|_{D} * f+\left.b w\right|_{D}=0$, that is $(b, e) \cdot W_{E}=0$, so $(b, e) \in \operatorname{Ker}\left(W_{E}\right)$.

Now if $e w=0$, that is $e=0$, then $F=\left\{F_{\alpha}=\left[\begin{array}{c}0 \\ v_{\alpha}\end{array}\right]\right\} \in H^{0}(C, K(-E))$ means $v=$ $\left\{v_{\alpha}\right\} \in H^{0}(C, K \otimes[-D])$, but we know that $H^{0}(C, K \otimes[-D])=\left\{w \in H^{0}(C, K)|w|_{D}=0\right\}$. Assume $v=b_{1} w_{1}+\cdots+b_{g} w_{g}=b w$, here $b=\left(b_{1}, \cdots, b_{g}\right)$, then $\left.b w\right|_{D}=0$ means $b W_{D}=0$, so $(b, 0) W_{E}=0$, this is $(b, 0) \in \operatorname{Ker}\left(W_{E}\right)$. That completes the proof.

From the proof, we get

Corollary 1. $H^{0}(C, K(-E)) \cong K \operatorname{er}\left(W_{E}\right)$, and in particular

$$
\operatorname{dim} H^{0}(C, K(-E))=2 g-\operatorname{rank}\left(W_{E}\right) .
$$

But from the definition of $W_{E}$, we know that

$$
\operatorname{dim} H^{0}(C, E)=d-\operatorname{rank}\left(W_{E}\right)+2 .
$$

We get the Riemann-Roch Theorem for base point free rank two vector bundle which generated by its sections:

Riemann-Roch Theorem. If $\mathrm{E}$ is a base point free rank two vector bundle which generated by its sections, then

$$
\operatorname{dim} H^{0}(C, E)-\operatorname{dim} H^{0}(C, K(-E))=\operatorname{deg}(E)-2(g-1) .
$$

Same as the case of line bundles, Riemann-Roch Theorem for all rank two vector bundles could be derived easily from this, we will not give it here.

Now, let $t \in T_{E}$, to get the dimension of $C_{2, d}^{r}$, we need to get the dimension of space $V=\left\{t \in T_{E} \mid B N^{*}(t) \in T_{B N(E)}\left(M_{d-r+1}\right)\right\}$. So we need to know under what condition $B N^{*}(t) \in T_{B N(E)}\left(M_{d-r+1}\right)$. From the theory of determinant variety, we know this same that 


$$
(b, e) B N^{*}(t)=(b, e)\left[\begin{array}{c}
\dot{W}_{D} *(-v) \\
\dot{W}_{D} *(-v) * f+W_{D} * u
\end{array}\right] \in \operatorname{Im}\left(W_{E}\right)
$$

for all $(b, e) \in \operatorname{Ker}\left(W_{E}\right)$. For this, we will first define a short exact sequence of sheaves.

Let $V$ be a vector bundle on $C$, we will use $V$ itself to denote the sheaf of holomorphic sections of $V$. For $E=\{I, f, D\}$, let $\left\{U_{\alpha}\right\}_{\alpha=1}^{k}$ be the given open cover of $C$, and $s=$ $\left\{s_{\alpha}\right\}_{\alpha=1}^{k} \in H^{0}(C,[D])$ be the canonical section of $[D]$, this is $s \in H^{0}(C,[D])$ and $\operatorname{div}(s)=D$. Let $f=\left\{f_{\alpha}\right\}$ be a given representation for $f \in H^{0}\left(C,\left.I\right|_{D}\right)$, where $f_{\alpha}$ is a holomorphic function on $U_{\alpha}$. Then by by using the transition matrix $E_{\alpha \beta}$ given in the proof of Lemma 4 , one can check directly that

$$
F=\left\{F_{\alpha}=\left[\begin{array}{l}
f_{\alpha} \\
s_{\alpha}
\end{array}\right]\right\} \in H^{0}(C, E) .
$$

is the lift of the canonical section $s$. Now let $P_{1}: K(-E) \mapsto K$ be the projective map which induced from sequence $0 \mapsto K[-D] \mapsto K \otimes[-E] \mapsto K \mapsto 0$, then from $F$ and $P_{1}$, we define a map of sheaves $K(-E) \mapsto K \oplus K$ by

$$
x \mapsto\left(P_{1}(x),-(x, F)\right)
$$

here $x \in K(-E)$, and $():, K(-E) \otimes E \mapsto K$ is the duality map. We also define a map of sheaves $\left.K \oplus K \mapsto K\right|_{D}$ to be $(s, t) \mapsto\left(\left.s\right|_{D} * f+\left.t\right|_{D}\right)$ for $(s, t) \in K \oplus K$.

Locally, let $\left\{U_{\alpha}\right\}$ be the given open cover of $C$, if $\left.\left[\begin{array}{l}a \\ b\end{array}\right] \in K(-E)\right|_{U_{\alpha}}$, then $K(-E) \mapsto K$ is defined by $\left[\begin{array}{l}a \\ b\end{array}\right] \mapsto\left(a,-a f_{\alpha}-b s_{\alpha}\right)$, and the map $K \oplus K \mapsto K_{D}$ could be given by $(c, d) \mapsto$ $\left(\left.c\right|_{D} * f+\left.d\right|_{D}\right)$.

Lemma 5 . The sequence $\left.0 \mapsto K(-E) \mapsto K \oplus K \mapsto K\right|_{D \mapsto 0}$ is a short exact sequence of sheaves on C.

Proof. We will use the local representation to give the proof.

If $\left[\begin{array}{l}a \\ b\end{array}\right] \in K(-E)$, and $\left[\begin{array}{l}a \\ b\end{array}\right] \mapsto(a,(-a f-b s))=0$, then $a=0$, and since $s \neq 0$ so $b s=0$ means $b=0$, the map $K(-E) \mapsto K \oplus K$ is injective.

If $(c, d) \in K \oplus K$, and $(c, d) \mapsto\left(\left.c\right|_{D} * f+\left.d\right|_{D}\right)=0$, we then get $\left.c\right|_{D} * f=-\left.d\right|_{D}$, by our Lemma $3, c$ can be lift locally to section of $K(-E)$ and same as Lemma 4 , $\left[\begin{array}{c}c \\ -(c f+d) / s\end{array}\right] \in K(-E)$ is one of the lift. But $\left[\begin{array}{c}c \\ -(c f+d) / s\end{array}\right] \mapsto(c,-c f+(c f+d))=(c, d)$. This shows that the sequence is exact at $K \oplus K$. proof.

Also it is easy to see that the map $\left.K \oplus K \mapsto K\right|_{D}$ is an onto map. This completes the

From this short exact sequence, we get a long exact sequence

$$
0 \mapsto H^{0}(C, K(-E)) \mapsto H^{0}(C, K \oplus K) \mapsto H^{0}\left(C,\left.K\right|_{D}\right) \mapsto H^{1}(C, K(-E)) \mapsto \cdots
$$

$a \in H^{0}\left(C,\left.K\right|_{D}\right)$ is in the image of map $H^{0}(C, K \oplus K)=H^{0}(C, K) \oplus H^{0}(C, K) \mapsto$ $H^{0}\left(C,\left.K\right|_{D}\right)$ if and only if $\delta(a)=0$, here $\delta: H^{0}\left(C,\left.K\right|_{D}\right) \mapsto H^{1}(C, K(-E))$ is the co-boundary map. But from Serra duality, we know that for $\delta(a) \in H^{1}(C, K(-E))$, $\delta(a)=0$ if and only if for any $f \in H^{0}(C, E)$, we have $(\delta(a), f)=0$. Here $($,$) :$ $H^{1}(C, K(-E)) \otimes H^{0}(C, E) \mapsto H^{1}(C, K)$ is the duality map.

Now assume, for open cover $\left\{U_{\alpha}\right\}, a$ is given by $a=\left\{a_{\alpha}\right\}$, where $a_{\alpha} \in H^{0}\left(U_{\alpha},\left.K\right|_{U_{\alpha}}\right)$ and $\left.a_{\alpha}\right|_{D \cap U_{\alpha}}=\left.a\right|_{D \cap U_{\alpha}}$. Then by direct calculation, we get $\delta(a) \in H^{1}(C, K(-E))$, could be represented as

$$
\delta(a)=\left\{\left[\begin{array}{c}
0 \\
k_{\alpha \beta}\left(-a_{\alpha}+a_{\beta}\right) / s_{\alpha}
\end{array}\right]\right\}=\left[\begin{array}{c}
0 \\
\tilde{\delta}(a)
\end{array}\right],
$$


where $\tilde{\delta}: H^{0}\left(C,\left.K\right|_{D}\right) \mapsto H^{1}(C, K[-D])$ is the co-boundary map from the following sequence

$$
0 \mapsto H^{0}(C, K[-D]) \mapsto^{\cdot s} H^{0}(C, K) \mapsto H^{0}\left(C,\left.K\right|_{D}\right) \mapsto H^{1}(C, K[-D]) \mapsto \cdots
$$

So for any $f=\left\{\left[\begin{array}{l}y_{\alpha} \\ x_{\alpha}\end{array}\right]\right\} \in H^{0}(C, E)$, the dual map could be given by

$$
\begin{gathered}
(\delta(a), f)=\left(\left\{\left[\begin{array}{c}
0 \\
\left(-a_{\alpha}+a_{\beta}\right) / s_{\alpha}
\end{array}\right]\right\},\left\{\left[\begin{array}{c}
y_{\alpha} \\
x_{\alpha}
\end{array}\right]\right\}\right) \\
\left.=\left\{\left(-a_{\alpha}+a_{\beta}\right) / s_{\alpha}\right) \cdot x_{\alpha}\right\}=\left(\tilde{\delta}(a),\left\{x_{\alpha}\right\}\right) .
\end{gathered}
$$

but $\delta(a)=0$ if and only if $(\delta(a), f)=0$ for all $f \in H^{0}(C, E)$, from what we get above, this is same that $\delta(a)=0$ if and only if for any $x=\left\{x_{\alpha}\right\} \in \operatorname{Im}\left\{H^{0}(C, E) \mapsto H^{0}(C,[D])\right\}$, $(\tilde{\delta}(a), x)=0$. We get the following Lemma.

Lemma 6. For $a \in H^{0}\left(C,\left.K\right|_{D}\right), \delta(a) \in H^{1}(C, K(-E))$, with $\delta(a)=0$ if and only if for any $x=\left\{x_{\alpha}\right\} \in \operatorname{Im}\left\{H^{0}(C, E) \mapsto H^{0}(C,[D])\right\},(\tilde{\delta}(a), x)=0$.

Now go back to the tangent map of $B N: H_{d} \mapsto M(2 g, d)$.

For $E=\{I, f, D\} \in C_{2, d}^{r}$, we know

$$
B N(E)=W_{E}=\left[\begin{array}{c}
W_{D} \\
W_{D} * f
\end{array}\right]
$$

if $t=(u,-v) \in T_{E}=H^{0}\left(C,\left.[D]\right|_{D}\right) \oplus H^{0}\left(C,\left.I\right|_{D}\right)$, then

$$
B N^{*}(t)=\left[\begin{array}{c}
\dot{W}_{D} *(-v) \\
\dot{W}_{D} *(-v) * f+W_{D} * u
\end{array}\right] .
$$

But we know that $B N^{*}(t) \in T_{B N(E)}\left(M_{d-r+1}\right)$ if and only if $\operatorname{Ker}\left(W_{E}\right) \cdot B N^{*}(t) \in$ $\operatorname{Im}\left(W_{E}\right)$. Since $\operatorname{Im}\left(W_{E}\right)=C^{2 g} \cdot W_{E}=\left\{(c, d)\left[\begin{array}{c}W_{D} \\ W_{D} * f\end{array}\right] \mid(c, d) \in C^{2 g}\right\}$. If we identify $C^{2 g}=C^{g} \oplus C^{g} \cong H^{0}(C, K) \oplus H^{0}(C, K)$, then we get

$$
\operatorname{Im}\left(W_{E}\right)=\operatorname{Im}\left\{H^{0}(C, K) \oplus H^{0}(C, K) \mapsto H^{0}\left(C,\left.K\right|_{D}\right)\right\} .
$$

Where the map $H^{0}(C, K) \oplus H^{0}(C, K) \mapsto H^{0}\left(C,\left.K\right|_{D}\right)$ is induced from above exact sequence.

From this we get $B N^{*}(t) \in T_{B N(E)}\left(M_{d-r+1}\right)$ if and only if for any $(b, e) \in \operatorname{Ker}\left(W_{E}\right)$, $(b, e) B N^{*}(t) \in \operatorname{Im}\left(W_{E}\right)$. This is $\delta\left((b, e) B N^{*}(t)\right)=0$. By Lemma 6, we get

Lemma 7. let $t \in T_{E}$, then $B N^{*}(t) \in T_{B N(E)}\left(M_{d-r+1}\right)$ if and only if for any $(b, e) \in$ $\operatorname{Ker}\left(W_{E}\right)$, we have $\left(\tilde{\delta}\left((b, e) B N^{*}(t)\right), x\right)=0$ for all $x \in \operatorname{Im}\left\{H^{0}(C, E) \mapsto H^{0}(C,[D])\right\}$.

But by direct calculation, we get

$$
\begin{gathered}
(b, e) B N^{*}(t)=(b, e)\left[\begin{array}{c}
\dot{W}_{D} *(-u) \\
\dot{W}_{D} *(-u) * f+W_{D} * v
\end{array}\right]=b \dot{W}_{D} * u+e \dot{W}_{D} * u * f+e W_{D} * v \\
=\left[\begin{array}{c}
e W_{D} \\
-\left(b \dot{W}_{D}+e \dot{W}_{D} * f\right)
\end{array}\right] *\left[\begin{array}{c}
u \\
-v
\end{array}\right] .
\end{gathered}
$$

Notice that by using local coordinate, it is easy to see that

$$
\left[\begin{array}{c}
e W_{D} \\
-\left(\dot{b\left(W_{D}\right)}+e \dot{W}_{D} * f\right.
\end{array}\right]=\left[\begin{array}{c}
e W_{D} \\
-\left(b W_{D}+e W_{D} * f\right) / s
\end{array}\right] .
$$

Since $(b, e) \in \operatorname{Ker}\left(W_{E}\right)$, by Lemma 4 , we get

$$
\left[\begin{array}{c}
e W_{D} \\
-\left(b W_{D}+e W_{D} * f\right) / s
\end{array}\right] \in \operatorname{Im}\left\{H^{0}(C, K(-E)) \mapsto H^{0}\left(C,\left.K(-E)\right|_{D}\right)\right\} .
$$


let it be the image of some $F \in H^{0}(C, K(-E))$. Now notice that $\left.E\right|_{D}=\left.\left.I\right|_{D} \oplus[D]\right|_{D}=T_{E}$ and $\left.K(-E)\right|_{D}=\left.\left.K\right|_{D} \oplus K[D]\right|_{D}=\left(\left.\left.I\right|_{D} \oplus[D]\right|_{D}\right)^{*}=T_{E}^{*}$ then follow the proof of Lemma 1.5 p162 [ACGH] step by step, for $x \in \operatorname{Im}\left\{H^{0}(C, E) \mapsto H^{0}(C,[D])\right\}$, we have

$$
\left(\tilde{\delta}\left((b, e) B N^{*}(t)\right), x\right)=(\tilde{\delta}(F * t), x)=\left(\delta_{1}(t),(F \otimes x)\right)=\left(t,\left.(F \otimes x)\right|_{D}\right)
$$

Where $\delta_{1}:\left(\left.\left.I\right|_{D} \oplus[D]\right|_{D}\right) \mapsto H^{1}(C, E[-D])$ is the co-boundary map follow from sequence $\left.0 \mapsto E[-D] \mapsto^{\cdot s} E \mapsto E\right|_{D} \mapsto 0$. So $t \in V=\left\{t \in T_{E} \mid B N^{*}(t) \in T_{B N(E)}\left(M_{d-r+1}\right)\right\}$ if and only if for any $F \in H^{0}(C, K(-E))$ and $x \in \operatorname{Im}\left\{H^{0}(C, E) \mapsto H^{0}(C,[D])\right\}$, we have $\left(t,\left.(F \otimes x)\right|_{D}\right)=0$. We get

Lemma 8. $t \in V=\left\{t \in T_{E} \mid B N^{*}(t) \in T_{B N(E)}\left(M_{d-r+1}\right)\right\}$, if and only if

$$
\begin{gathered}
t \in\left\{\operatorname { I m } \left\{H^{0}(C, K(-E)) \otimes \operatorname{Im}\left\{H^{0}(C, E) \mapsto H^{0}(C,[D])\right\}\right.\right. \\
\left.\mapsto H^{0}(C, K(-E)[D]) \mapsto H^{0}\left(C,\left.K(-E)[D]\right|_{D}\right)\right\}^{\perp} .
\end{gathered}
$$

Now assume $E \in C_{2, d}^{r}-C_{2, d}^{r+1}$,From what we get above, the expected dimension of $C_{2, d}^{r}$ at $E$ could be given by

$$
\begin{gathered}
\operatorname{dim}\left(C_{2, d}^{r}\right)=\operatorname{dim}(V)= \\
2 d-\operatorname{dim}\left\{\operatorname { I m } \left\{H^{0}(C, K(-E)) \otimes \operatorname{Im}\left\{H^{0}(C, E) \mapsto H^{0}(C,[D])\right\}\right.\right. \\
\left.\left.\mapsto H^{0}(C, K(-E)[D]) \mapsto H^{0}\left(C,\left.K(-E)[D]\right|_{D}\right)\right\}\right\}= \\
2 d-(2(g-1)-d+r+1) r+2(g-1)-d+r+1+\operatorname{dim} W .
\end{gathered}
$$

where $(2(g-1)-d+r+1) r=\operatorname{dim}\left[H^{0}(C, K(-E)) \otimes \operatorname{Im}\left\{H^{0}(C, E) \mapsto H^{0}(C,[D])\right\}\right]=$ $\operatorname{dim} H^{0}(C, K(-E)) \times \operatorname{dimIm}\left\{H^{0}(C, E) \mapsto H^{0}(C,[D])\right\}$, and $2(g-1)-d+r+1=$ $\operatorname{dimKer}\left\{H^{0}(C, K(-E)[D]) \mapsto H^{0}(C, K(-E)[D] \mid D)\right\}, W=\operatorname{Ker}\left\{H^{0}(C, K(-E)) \otimes\right.$ $\left.\operatorname{Im}\left\{H^{0}(C, E) \mapsto H^{0}(C,[D])\right\} \mapsto H^{0}(C, K(-E)[D])\right\}$.

We then get

$$
\begin{aligned}
\operatorname{dim}\left(C_{2, d}^{r}\right)=4(g-1)+ & 1-(r+1)(2(g-1)-d+r+1)+2 r+1+\operatorname{dim} W \\
& =\rho(2, d, r)+2 r+1+\operatorname{dim} W
\end{aligned}
$$

Theorem 3. $C_{2, d}^{r}$ has the expected dimension $\rho(2, d, r)+2 r+1$ at $E \in C_{2, d}^{r}-C_{2, d}^{r+1}$, if and only if for all $E \in C_{2, d}^{r}, W=\{0\}$.

This is the same that $C_{2, d}^{r}$ has the expected dimension $\rho(2, d, r)+2 r+1$, if and only if for all $E \in C_{2, d}^{r}$, the map

$$
H^{0}(C, K(-E)) \otimes \operatorname{Im}\left\{H^{0}(C, E) \mapsto H^{0}(C,[D])\right\} \mapsto H^{0}(C, K(-E)[D])
$$

is injective.

Compare with the case of line bundles, we then called the map

$$
H^{0}(C, K(-E)) \otimes \operatorname{Im}\left\{H^{0}(C, E) \mapsto H^{0}(C,[D])\right\} \mapsto H^{0}(C, K(-E)[D])
$$

the Petri map for rank two vector bundles. We have

Theorem 4. $C_{2, d}^{r}$ has the expected dimension $\rho(2, d, r)+2 r+1$, if and only if for all $E \in C_{2, d}^{r}$, the Petri map is injective.

This is a generalization of Lemma 1.6 of $[\mathrm{ACGH}] \mathrm{P} 163$. 


\section{REFERENCES}

[ACGH] Arbarello, E., Cornaba, M., Griffiths, P., Harris, J., Geometry of Algebraic curves, Vol I, Springer-Verlag, N.Y. 1984.

[A] AтזYан, M.F., Vector Bundles on Elliptic Curves, Proc London Math Soc, 7 (1957), pp. 414-452.

[G] Gieseker, D., Stable curves and special divisors, Invent Math., 66 (1982), pp. 25-275.

[GH] Griffiths, P., HARRIS, J., The dimension of the variety of special linear systems on a general curve, Duke Math. J, 47 (1980), pp. 233-272.

[LN] Lange, H., Narasimhan, M.S., Maximal Sub-bundles of Rank Two Bundles on Curves, Math Ann, 266 (1983), pp. 55-72.

[T] TAN, X.J., Some results on the existence of rank two special stable vector bundles, Manuscripta Math, 75 (1992), pp. 365-373.

[TE] TeIxidor i Bigas, M., On the Gieseker-Petri map for rank two vector bundles, Manuscripta Math, 75 (1992), pp. 375-382. 